\title{
Global sensor selection for maneuvering target tracking in clutter
}

\author{
Wenling Li \\ The Seventh Research Division, Beihang University \\ 37 Xueyuan Road, Haidian District, Beijing, 100191, China \\ Yingmin Jia \\ The Seventh Research Division, Beihang University \\ 37 Xueyuan Road, Haidian District, Beijing, 100191, China \\ E-mail:lwlmath@buaa.edu.cn,ymjia@buaa.edu.cn
}

\begin{abstract}
This paper studies the problem of sensor selection for maneuvering target tracking in the cluttered environment. By modeling the target dynamics as jump Markov linear systems, a decentralized tracking algorithm is developed by applying the extended Kalman filter and the probabilistic data association technique. A cost function that minimizes the expected filtered mean square position error is utilized and a sensor selection scheme is proposed. A numerical example is provided to illustrate the effectiveness of the proposed approach.
\end{abstract}

Keywords: Sensor selection, Jump Markov system, Extended Kalman filter, Maneuvering target tracking, Clutter

\section{Introduction}

Sensor or node selection has been found a wide range of applications in diverse civilian and military areas such as sensor placement for structures, ${ }^{1}$ chemical plant control $^{2}$ and wireless networks. ${ }^{3,4}$ In recent years the problem of sensor selection for target tracking has been investigated. As pointed out in Ref. 5, the main idea beyond sensor selection is to optimize a cost function that represents the localization accuracy constrained by the number of active sensors. A cost function that minimizes the geometrical dilution of precision (GDOP) is used to derive the sensor selection scheme where the best three sensors are selected. Based on the GDOP measure, an adaptive sensor selection strategy has been proposed in which the signal power measurements are used. By combining with the prior information, which is not accounted for the GDOP measure, global sensor selection method that minimizes the expected filtered mean square position error have been studied for bearing-only tracking. Especially, two strategies including the 'add one sensor node at a time' and the 'simplex' have been developed for selecting the best subset of active sensors. ${ }^{6}$ These results are extended to propose local sensor selection schemes which do not require the knowledge of all the sensors locations in the networks.

In the target tracking community, the single model approach is used mainly for targets with fixed kinematic behaviors while for targets with multiple kinematics behaviors (i.e., maneuvering targets), the multiple model tracking algorithm is preferred. ${ }^{7}$ The problem of sensor selection for maneuvering target tracking has been investigated in Ref. 8 and 9. To our knowledge, little research has been done to deal with the problem of 
sensor selection for target tracking in the cluttered environment.

In this paper, we consider the problem of sensor selection for maneuvering target tracking in the cluttered environment. By using the global knowledge of all sensors locations, a cost function that minimizes the expected filtered mean square localization error is developed to select a given number of active sensors. As the cost function is derived based on the decentralized structure, we investigate how to derive the decentralized fusion formula by applying the basic extended Kalman filter (EKF) and the PDA technique so that the clutter information can be combined into the cost function. Meanwhile, we have to circumvent the difficulty encountered in multiple model decentralized fusion. ${ }^{10}$ By minimizing the cost function, a sensor selection scheme is proposed based on the add one sensor node at a time' strategy. Simulations are provided to illustrate the effectiveness of the proposed algorithm.

\section{Problem formulation}

In this paper, we consider a two-dimensional (2-D) target-observer scenario. The target dynamics is modeled as the following coordinated turn model

$$
x_{k+1}=\left[\begin{array}{cccc}
1 & 0 & \frac{\sin (\omega T)}{\omega} & -\frac{1-\cos (\omega T)}{\omega} \\
0 & 1 & \frac{1-\cos (\omega T)}{\omega} & \frac{\sin (\omega T)}{\omega} \\
0 & 0 & \cos (\omega T) & \sin (\omega T) \\
0 & 0 & \sin (\omega T)_{T} & \cos (\omega T)
\end{array}\right] x_{k}+w_{k}
$$

where $x_{k}=\left(x_{k}, y_{k}, \dot{x}_{k}, \dot{y}_{k}\right)^{T}$ denotes the target state. $\left(x_{k}, y_{k}\right)$ and $\left(\dot{x}_{k}, \dot{y}_{k}\right)$ represent the target position and velocity components, respectively. $\omega$ denotes the turn rate and $T$ is the sampling time period. $\omega_{k}$ is zero-mean white Gaussian noise with covariance $\mathrm{Q}_{k}$.

To formulate the target dynamics into the framework of jump Markov systems, we assume that at any time the target motion obeys one of $\mathrm{M}$ dynamic behavior models, which can be described by the above coordinated turn model with different turn rate. The switching between models is governed by a timehomogeneous first-order Markov chain $r_{k}$ with known transition probability $\pi_{i j}=P\left\{r_{k}=j \mid r_{k-1}=i\right\}$.

A radio receiver that provides the signal power measurement is used throughout this paper. The target- originated power measurement from sensor $s$ can be modeled in a logarithmic form

$$
\begin{aligned}
& z_{k, s}=K-10 \eta \log _{10}\left(r_{k, s}\right)+v_{k, s} \\
& r_{k, s}=\sqrt{\left(x_{k}-x_{s}\right)^{2}+\left(y_{k}-y_{s}\right)^{2}}, \quad s=1,2, \cdots, N
\end{aligned}
$$

where $\mathrm{K}$ and $\eta \in[2,5]$ denote the transmission power and the path loss exponent, respectively. They are dependent on the radio environment, the antenna characteristics and so on. $\left(x_{s}, y_{s}\right)$ represents the position of sensor $S$ and $r_{k, s}$ is the relative distance between sensor $S$ and the target. $\mathrm{N}$ is the number of sensors. $v_{k, s}$ is zero-mean white Gaussian with variance $R_{k, s}$.

Our aim is to find the set of active sensors that optimizes the cost function. As the clutter is considered, we should investigate how to combine the clutter information into the cost function.

\section{Sensor election for Maneuvering Target Tracking in Clutter}

\section{Decentralized fusion of EKF-PDA}

Assume that the target dynamics and the measurement model can be represented by

$$
\begin{gathered}
x_{k+1}=\Phi_{k} x_{k}+w_{k} \\
z_{k, s}=h_{s}\left(x_{k}\right)+v_{k, s}, s=1,2, \cdots, N
\end{gathered}
$$

where $\Phi_{k}$ and the covariance $\sum_{k}$ of $w_{k}$ are derived by the best-fitting Gaussian approximation. $h_{s}$ is the nonlinear measurement function of sensor $S$. By combining the EKF and the PDA technique, a single forward run of the tracking algorithm proceeds as follows for each sensor s.

First of all, the predicted mean and covariance are obtained by using a standard Kalman filter

$$
\begin{aligned}
& \hat{x}_{k \mid k-1}^{s}=\Phi_{k-1} \hat{x}_{k-1 \mid k-1}^{s} \\
& P_{k \mid k-1}^{s}=\Phi_{k-1} P_{k-1 \mid k-1}^{s} \Phi_{k-1}^{T}+\Sigma_{k-1}
\end{aligned}
$$

Then, the predicted measurement and the innovation covariance can be derived as

$$
\begin{gathered}
\hat{z}_{k \mid k-1}^{s}=h_{s}\left(\hat{x}_{k \mid k-1}^{s}\right) \\
v_{k, s}^{i}=z_{k, s}^{i}-\hat{z}_{k \mid k-1}^{i} \\
S_{k, s}=H_{k, s} P_{k \mid k-1}^{s} H_{k, s}^{T}+R_{k, s}
\end{gathered}
$$

where $H_{k, s}$ is the Jacobian matrix of the nonlinear function $h_{s}$ evaluated at $\hat{x}_{k-1 \mid k-1}^{s}$. 
After receiving the measurements $Z_{k, s}$ by sensor s, $z_{k, s}^{i}$ is validated if

$$
\left[z_{k, s}^{i}-\hat{z}_{k \mid k-1}^{s}\right]^{T} S_{k, s}^{-1}\left[z_{k, s}^{i}-\hat{z}_{k \mid k-1}^{s}\right]<\gamma
$$

where $\gamma$ is the gate threshold.

By assuming that the number of clutter in the validated region is assumed to be Poisson distributed, a parametric PDA technique is used to generate the association event probability

$$
\begin{gathered}
\beta_{k, s}^{0}=\frac{b_{k, s}}{b_{k, s}+\sum_{j=1}^{m_{k, 2}} d_{k, s}^{j}} \\
\beta_{k, s}^{i}=\frac{b_{k, s}}{b_{k, s}+\sum_{j=1}^{m_{k, 2}} d_{k, s}^{j}}, i=1, \cdots, m_{k, s}
\end{gathered}
$$

Where

$$
\begin{gathered}
d_{k, s}^{i}=\exp \left(-0.5\left[v_{k, s}^{i}\right]^{T} S_{k, s}^{-1} v_{k, s}^{i}\right) \\
b_{k, s}=\lambda\left|2 \pi S_{k, s}\right|^{1 / 2} \frac{1-P_{D} P_{G}}{P_{D}}
\end{gathered}
$$

$P_{D}$ is the detection probability, $P_{G}$ is the probability that the measurement originated from the target falls into the validated region, and $\lambda$ is the spatial density of clutter. Finally, the updated estimates can be computed by

$$
\begin{gathered}
\hat{x}_{k \mid k}^{s}=\hat{x}_{k \mid k-1}^{s}+K_{k, s} v_{k, s} \\
\hat{P}_{k \mid k}^{s}=\hat{P}_{k \mid k-1}^{s}-K_{k, s}\left(S_{k, s}-B_{k, s}\right) K_{k, s}^{T}
\end{gathered}
$$

where

$$
\begin{gathered}
v_{k, s}=\sum_{i=1}^{m_{k, 2}} \beta_{k, s}^{i} v_{k, s}^{i} \\
K_{k, s}=P_{k \mid k-1}^{s} H_{k, s}^{T} S_{k, s}^{-1} \\
B_{k, s}=\beta_{k, s}^{0} S_{k, s}+\sum_{i=1}^{m_{k, 2}} \beta_{k, s}^{i} v_{k, s}^{i}\left[v_{k, s}^{i}\right]^{T}-v_{k, s} v_{k, s}^{T}
\end{gathered}
$$

In order to develop the decentralized fusion formula, we would like to rewrite the updated mean and covariance as the same form of the usual Kalman filter, i.e.,

$$
\begin{gathered}
\hat{x}_{k \mid k}^{s}=\hat{x}_{k \mid k-1}^{s}+\widetilde{K}_{k, s}\left(\widetilde{z}_{k, s}-\hat{z}_{k \mid k-1}^{s}\right) \\
P_{k \mid k}^{s}=P_{k \mid k-1}^{s}-\widetilde{K}_{k, s} \widetilde{S}_{k, s} \widetilde{K}_{k, s}^{T} \\
\widetilde{\mathrm{K}}_{k, s}=P_{k \mid k-1}^{s} H_{k, s}^{T} \widetilde{S}_{k, s}^{-1} \\
\widetilde{S}_{k, s}=H_{k, s} P_{k \mid k-1}^{s} H_{k, s}^{T}-\widetilde{R}_{k, s}
\end{gathered}
$$

It can be observed that our aim is to define $\widetilde{z}_{k, s}$ and $\widetilde{R}_{k, s}$.To this end, we have

$$
\begin{aligned}
& \widetilde{K}_{k, s} \widetilde{S}_{k, s} \widetilde{K}_{k, s}^{T}=P_{k \mid k-1}^{s} H_{k, s}^{T} \widetilde{S}_{k, s}^{-1} H_{k, s} P_{k \mid k-1}^{s} \\
& =K_{k, s} S_{k, s} \widetilde{S}_{k, s}^{-1} S_{k, s} K_{k, s}^{T}
\end{aligned}
$$

$$
\begin{aligned}
& \widetilde{S}_{k, s}=\left(S_{k, s}^{-1}-S_{k, s}^{-1} B_{k, s} S_{k, s}^{-1}\right)^{-1} \\
& =S_{k, s}-S_{k, s} S_{k, s}^{-1}\left(S_{k, s}^{-1} S_{k, s} S_{k, s}^{-1}-B_{k, s}^{-1}\right)^{-1} S_{k, s}^{-1} S_{k, s} \\
& =S_{k, s}+\left(B_{k, s}^{-1}-S_{k, s}^{-1}\right)^{-1}
\end{aligned}
$$

Therefore,

$$
\widetilde{R}_{k, s}=R_{k, s}+\left(B_{k, s}^{-1}-S_{k, s}^{-1}\right)^{-1}
$$

On the other hand, we have

$$
K_{k, s} v_{k, s-} \widetilde{K}_{k, s}\left(\widetilde{z}_{k, s}-\hat{z}_{k \mid k-1}^{s}\right)=0
$$

which leads to

$$
\widetilde{z}_{k, s}=\left[I+\left(B_{k, s}^{-1}-S_{k, s}^{-1}\right)^{-1} S_{k, s}^{-1}\right] v_{k, s}+\hat{z}_{k \mid k-1}^{s}
$$

Based on the above EKF-PDA tracking algorithm, we can obtain the decentralized fusion formula. We assume that each sensor knows its position and they share the common prior information. Suppose each active sensor have obtained estimates $\hat{x}_{k-1 \mid k-1}$ and $P_{k-1 \mid k-1}$ at time step $\mathrm{k}-1$, the filtered estimates can be derived by each sensor via communicating with other active sensors

$$
\begin{gathered}
\hat{x}_{k \mid k-1}=\Phi_{k-1} \hat{x}_{k-1 \mid k-1} \\
P_{k \mid k-1}=\Phi_{k} P_{k-1 \mid k-1} \Phi_{k}^{T}+\Sigma_{k-1} \\
\hat{x}_{k \mid k}=P_{k \mid k}\left[P_{k \mid k-1}^{-1} \hat{x}_{k \mid k-1}+\sum_{s \in N_{a}} H_{k, s}^{T} \widetilde{R}_{k, s}^{-1}\left[\widetilde{z}_{k, s}-h_{s}\left(\hat{x}_{k \mid k-1}\right)+H_{k, s} \hat{x}_{k \mid k-1}\right]\right] \\
P_{k \mid k}^{-1}=P_{k \mid k-1}^{-1}+\sum_{s \in N_{a}} H_{k, s}^{-1} \widetilde{R}_{k, s}^{-1} H_{k, s}
\end{gathered}
$$

\section{Sensor selection}

When the signal power measurement is applied, the updated covariance for the decentralized fusion of EKFPDA can be rewritten as

$$
J_{f}=J_{p}+\left[\begin{array}{l}
J, 0 \\
0,0
\end{array}\right]
$$

Where $J_{f}=P_{k \mid k}^{-1}, J_{p}=P_{k \mid k-1}^{-1}$ and

$$
J=\left(10 \eta \log _{10} e\right)^{2} \sum_{s \in N_{a}} \widetilde{R}_{k, s}^{-1} r_{k, s}^{-2}\left[\begin{array}{l}
\cos ^{2} \phi_{k, s}, \sin \phi_{k, s} \cos \phi_{k, s} \\
\sin \phi_{k, s} \cos \phi_{k, s}, \sin ^{2} \phi_{k, s}
\end{array}\right]
$$

Note that

$$
H_{k, s}=-10 \eta \log _{10} e\left[r_{k, s}^{-1} \cos \phi_{k, s}, r_{k, s}^{-1} \sin \phi_{k, s}, 0,0\right]
$$

where $\left(r_{k, s}, \phi_{k, s}\right)$ is the position of sensor s relative to the predicted target position in polar coordinates.

The aim of sensor selection is to search for the minimum expected mean square posterior error which can be expressed in terms of the posterior Fisher information matrix. Specifically, the cost function to be minimized for sensor selection is

$$
\rho\left(\mathrm{N}_{a}\right)=\left[J_{f}^{-1}\right]_{1,1}+\left[J_{f}^{-1}\right]_{2,2}
$$


Where $[A]_{i, j}$ denotes the $(i, j)$ th element of the matrix A

$$
\rho\left(\mathrm{N}_{a}\right)=\frac{\operatorname{tr}\left\{\tilde{J}_{p}\right\}+\operatorname{tr}\{J\}}{\left|\tilde{J}_{p}+J\right|}=\frac{\operatorname{tr}\left\{\tilde{J}_{p}\right\}+\operatorname{tr}\{J\}}{\left|\tilde{J}_{p}\right|+|J|+J_{k}^{d}}
$$

where ' $\operatorname{tr}$ ' represents the trace of the matrix, and

$$
\begin{aligned}
& \widetilde{J}_{p}=\left[J_{p}\right]_{1: 2,1-2}-\left[J_{p}\right]_{1: 2,3: 4}\left(\left[J_{p}\right]_{3: 4,3: 4}\right)^{-1}\left[J_{p}\right]_{3: 4,1: 2} \\
& J_{k}^{d}=\left(10 \eta \log _{10} e\right)^{2} \sum_{s \in N_{a}}\left[\left[\widetilde{J}_{p}\right]_{1,1} \widetilde{R}_{k, s}^{-1} r_{k, s}^{-2} \sin ^{2} \phi_{k, s}\right. \\
& \left.+\left[\widetilde{J}_{p}\right]_{2,2} \widetilde{R}_{k, s}^{-1} r_{k, s}^{-2} \cos ^{2} \phi_{k, s}-2\left[\widetilde{J}_{p}\right]_{1,2} \widetilde{R}_{k, s}^{-1} r_{k, s}^{-2} \sin \phi_{k, s} \cos \phi_{k, s}\right]
\end{aligned}
$$

A practical strategy called as 'add one sensor node at a time'. For this strategy, an initial active set is established by using the exhaustive search, which includes two active sensor nodes and requires evaluating $\rho\left(N_{a}\right)$ over $N(N-1) / 2$ configurations. Then the rest of sensor nodes are added to the initial active set one at a time by minimizing $\rho\left(N_{a}\right)$.

\section{Simulations}

Three models corresponding to different turn rates are used. The sampling period is taken to be $1 \mathrm{~s}$ for target motion model. The network consists of 30 sensors randomly scattered over a field. The sensing range for each sensor is $25 \mathrm{~m}$. For the signal power measurements, the transmission power is taken to be $9 \mathrm{dBm}$ and the path loss exponent is 3 . The variance of the measurement noise is 0.2 for all sensors. The detection probability and the gate probability are set to 0.997 and 0.999 , respectively. The gate threshold is 16 and the spatial density of clutter is 0.1 .

In the simulations, the root mean square error (RMSE) in position is used for performance comparison. The RMSE in position of the proposed algorithm with different numbers of active sensors is shown in Fig.1. It can be observed that the tracking performance is improved as the number of active sensors is used and the best performance is achieved when all the active sensors in the sensing range are utilized.

\section{Conclusion}

In this paper, we propose a global sensor selection scheme for tracking maneuvering target in the cluttered environment. A formulation of PDA for decentralized fusion is derived which helps the definition of the cost function for sensor selection. This is implemented by developing novel measurement vector and noise covariance matrix combining the clutter information. Simulation results are provided to verify that the proposed algorithm.

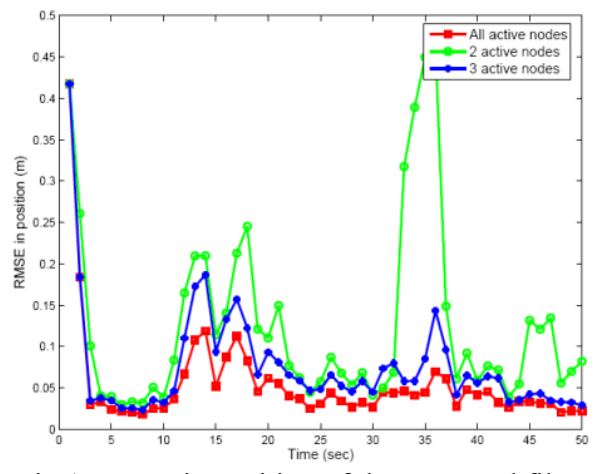

Fig.1 RMSE in position of the proposed filter.

\section{References}

1. D. Kammer, Sensor placement for on-orbit modal identification and correlation of large space structures, Journal of Guidance, Control, and Dynamics, 14(2) (1991) 251-259.

2. K. Kookos and J. Perkins, A systematic method for optimum sensor selection in inferential control systems, Industrial Engineering Chemistry Research, 38(11) (1999) 4299-4308.

3. F. Zhao and L. Guibas, Wireless Sensor Networks: An Information Processing Approach. (Morgan Kaufmann, San Mateo, 2004).

4. H. Rowaihy, et al., A survey of sensor selection schemes in wireless sensor networks, Proceedings of the SPIE, (2007), 6562-65621.

5. L. M. Kaplan, Global node selection for localization in a distributed sensor network, IEEE Transactions on Aerospace and Electronic Systems, 42(1) (2006) 113-135

6. L. M. Kaplan, Local node selection for localization in a distributed sensor network, IEEE Transactions on Aerospace and Electronic Systems, 42(1) (2006) 136-146.

7. X. R. Li and V. P. Jilkov, Survey of maneuvering target tracking. Part $\mathrm{V}$ : multiple-model methods, IEEE Transactions on Aerospace and Electronic Systems, 41(4) (2005) 1255-1321.

8. Q. Le, et al., Multiple-mode Kalman filtering with node selection using bearings-only measurements, Proceedings of the 36th IEEE Southeastern Symposium on System Theory, (USA ,Atlanta, 2004), pp.185-189.

9. S. Zhang et al., IMM filter based sensor scheduling for maneuvering target tracking in wireless sensor networks, Proceedings of the 3rd International Conference on Intelligent Sensors, Sensor Networks and Information, (Melbourne, Qld, 2007), pp. 287-292.

10. W. L. Li and Y. M. Jia, Distributed interacting multiple model $\mathrm{H} \infty$ filtering fusion for multiplatform maneuvering target tracking in clutter, Signal Processing, 90(5) (2010) 1655-1668. 